Artículo original

\title{
Sobre la dinámica de una partícula en rotación usando el concepto de invariancia gauge
}

\author{
Mauricio Rozo-Clavijo \\ Universidad Pedagógica Nacional, Departamento de Física, Bogotá, Colombia
}

\begin{abstract}
Resumen
Se hace una descripción del movimiento de una partícula en rotación haciendo uso del plano complejo y utilizando procedimientos similares al de la construcción de una teoría gauge local. El análisis permite describir el comportamiento de la partícula partiendo de la exigencia de la invariancia gauge de la ecuación de posición. Invariancia que al ser exigida conduce a la introducción de un parámetro compensatorio que se interpreta como la velocidad angular de la partícula. A partir del análisis se obtienen los efectos dinámicos que determinan su movimiento, los cuales son equivalentes a los obtenidos desde una descripción hecha respecto a un sistema de referencia en rotación haciendo uso de coordenadas polares. Este análisis presenta una manera novedosa de deducir las ecuaciones dinámicas de una partícula en rotación, lo cual representa un aporte original y pedagógico para la enseñanza de la física. (C) 2016. Acad. Colomb. Cienc. Ex. Fis. Nat.
\end{abstract}

Palabras clave: Enseñanza; Invariancia gauge; Efectos dinámicos; Parámetro compensador; Transformación gauge.

On the dynamics of a rotating particle using the concept of gauge invariance

\begin{abstract}
A description is performed on the dynamics of a particle in rotational motion, using a complex plane and similar procedures to those implemented in the construction of a local gauge theory. The analysis allows the description of the behavior of the particle, by requiring gauge invariance in its equation of position. Requiring gauge invariance leads to the addition of an extra term in the equation, which is interpreted as the angular velocity of the particle. From this analysis, the dynamical effects that determine the motion of the particle are obtained, which are equivalent to those obtained by describing the rotating system using polar coordinates. This kind of analysis is important since it presents a new method to obtain the equations that describe the dynamics of a particle in rotational motion. (C) 2016. Acad. Colomb. Cienc. Ex. Fis. Nat.
\end{abstract}

Key words: Teaching; Gauge invariance; Dynamic effects; Compensatory parameter; Gauge transformation

\section{Introducción}

En la descripción del movimiento de una partícula en rotación se utiliza en general un sistema de coordenadas polar, aunque se puede utilizar cualquier sistema de coordenadas, este sistema permite simplificar el problema (Kleppner, $\boldsymbol{e t}$ al., 1973) y (Feynman, 1964). Haciendo uso del sistema de coordenadas polar y tomando en consideración las llamadas fuerzas inerciales se obtienen los efectos dinámicos de la partícula en rotación como son: la fuerza centrífuga $\vec{F}=m \ddot{r} \hat{r}$, la fuerza tangencial $\vec{F}=m r \ddot{\theta} \hat{\theta}$, la fuerza centrípeta $\vec{F}=$ $-m r \dot{\theta}^{2} \hat{r}$ y la fuerza de coriolis $\vec{F}=2 m \dot{r} \dot{\theta} \hat{\theta}$ (Marion, 1992) y (Goldstein, 1987) las cuales se pueden escribir de la siguiente forma:

$$
\vec{F}=m\left(\ddot{r}-r \dot{\theta}^{2}\right) \hat{r}+m(r \ddot{\theta}+2 \dot{r} \dot{\theta}) \hat{\theta}
$$

Por otro lado, las simetrías han jugado un rol importante en física partiendo desde las simetrías del espacio-tiempo en relatividad hasta las simetrías gauge. En general, las simetrías han fijado una ruta en la construcción de explicaciones a los hechos y cosas que suceden en el mundo físico. En este sentido, se ha construido un esquema sobre la interacción entre partículas denominado: teoría gauge no abeliana con simetría local, (Greiner, 2000) y (Quigg, 1993). Qué significa tan rimbombante nombre constituye una motivación en el presente trabajo.

En el esquema de la teoría gauge se hace uso del formalismo lagrangiano, que permite por un lado, determinar la dinámica de la interacción al exigir que el lagrangiano sea invariante bajo un grupo de simetría gauge, y por otro, se establece que el lagrangiano sea invariante bajo una transformación de Lorentz. Lo anterior sugiere que la invariancia gauge del lagrangiano puede ser considerada como un principio dinámico (Novaes, 2000), en tanto que

\footnotetext{
Correspondencia:

Mauricio Rozo-Clavijo, mclavijo@pedagogica.edu.co

Recibido: 11 de julio de 2016
}

Aceptado: 28 de octubre de 2016 
la invariancia del lagrangiano bajo la transformación de Lorentz garantiza que las predicciones que surjan de la teoría sean invariantes.

Por lo tanto, la simetría gauge juega un papel fundamental en la descripción de las interacciones ya que a partir de ésta se predice la forma como las partículas interactúan, lo cual constituye una motivación para abordar el concepto de invariancia gauge desde otro contexto, con el fin de aportar una metodología o ruta de trabajo que facilite una mejor comprensión de la idea gauge por parte de los estudiantes.

Ya que el concepto de invariancia gauge, local y global, resulta generalmente abstracto a los estudiantes, se propone implementar la invariancia gauge dentro del contexto de la mecánica newtoniana y en especial en una partícula con un movimiento de rotación, con el propósito de utilizar procedimientos similares al de la construcción de una teoría gauge local, y así obtener los efectos dinámicos de la partícula. Desde este contexto, se parte de la exigencia de la invariancia gauge de la ecuación de posición de la partícula; invariancia que al ser exigida conduce a obtener los efectos dinámicos.

Bajo esta perspectiva, se analiza el movimiento de la partícula en rotación haciendo uso del plano complejo para dar la posición $(z)$ de la partícula e implementar el concepto de invariancia gauge. A partir de este análisis se obtienen los efectos dinámicos como son: las fuerzas de coriolis, centrífuga, centrípeta y tangencial (Goldstein, 1987). Finalmente, la implementación del concepto de invariancia gauge dentro de este contexto, permite por un lado, humanizar este concepto ya que es de difícil comprensión por parte de los estudiantes, y por otro, acerca a los estudiantes a las ideas contemporáneas de la física.

Sobre la idea Gauge. La idea gauge como principio dinámico fue por primera vez apreciada por Hermann Weyl motivado por encontrar una base geométrica que diera cuenta de las interacciones gravitacional y electromagnética. En su artículo "Gravitation and electricity" (Weyl, 1918), propone por primera vez la idea gauge "guiding field" (Weyl, 1918) y (Weyl, 1952) para unificar la fuerza gravitacional y electromagnética hasta entonces conocidas (1900). Su propuesta se fundamenta en una geometría que explique no sólo los fenómenos gravitatorios sino también aquellos que están relacionados con campos electromagnéticos dentro del contexto de una teoría de campo unificado de la gravedad y el electromagnetismo.

Weyl propone para esto una generalización del concepto de medida, argumentando que las medidas físicas son relativas y lo que tiene significado físico es el intervalo,

$$
\Delta S^{2}=\left(x_{2}-x_{1}\right)^{2}+\left(y_{2}-y_{1}\right)^{2}+\left(z_{2}-z_{1}\right)^{2} \text {. }
$$

El intervalo medido por un observador entre dos eventos (puntos) en un espacio de tres dimensiones, podrá ser calculado de diferentes formas por los observadores, es decir, la posición inicial y final de cada evento podrá ser recalibrada de diferentes formas manteniendo el intervalo de la distancia entre los dos puntos invariante para todos los observadores.
Bajo este contexto, los eventos que ocurren en cada punto del espacio quedan determinados mediante las coordenadas $\vec{r}_{i}=\vec{r}_{i}\left(x_{1}, y_{1}, z_{1}\right)$ y $\vec{r}_{f}=\vec{r}_{f}\left(x_{2}, y_{2}, z_{2}\right)$ respectivamente, las cuales pueden ser recalibradas adicionando una constante arbitraria de la siguiente forma:

$$
\begin{aligned}
& \vec{r}_{i} \rightarrow \vec{r}_{i}^{\prime}=\vec{r}_{i}+\text { cte } ; \\
& \vec{r}_{f} \rightarrow \vec{r}_{f}^{\prime}=\vec{r}_{f}+\text { cte. }
\end{aligned}
$$

Lo anterior significa que la posición inicial y final de los eventos no está unívocamente determinada. La medición de la posición de un evento en cada punto del espacio puede ser arbitraria respecto a cada observador. A un mismo intervalo de distancia pueden corresponder diferentes posiciones, inicial y final, dependiendo de la constante arbitraria que se adicione. Es fácil de comprobar que el intervalo no cambia al sustituir la transformación (3) en (2). La transformación de coordenadas (3) no cambia el intervalo de la distancia entre los dos eventos, lo cual significa, que es el intervalo $(\Delta S)$ de la magnitud considerada lo que tiene significado físico. Esta invariancia es denominada invariancia gauge global del intervalo entre dos eventos, ya que la constante que se adiciona es igual para todos los observadores.

Existe otra manera de realizar la recalibración de la posición de un evento en un punto del espacio. En este caso, cada observador puede adicionar una constante diferente a la posición inicial y final de los eventos sin que el intervalo de la distancia $(\Delta S)$ cambie, es decir, el intervalo queda invariante bajo una transformación local,

$$
\begin{aligned}
& \vec{r}_{1 i} \rightarrow \vec{r}_{1 i}^{\prime}=\vec{r}_{1 i}+c_{1} ; \\
& \vec{r}_{1 f} \rightarrow \vec{r}_{1 f}^{\prime}=\vec{r}_{1 f}+c_{1}, \\
& \text { ó } \\
& \vec{r}_{2 i} \rightarrow \vec{r}_{2 i}^{\prime}=\vec{r}_{2 i}+c_{2} ; \\
& \vec{r}_{2 f} \rightarrow \vec{r}_{2 f}^{\prime}=\vec{r}_{2 f}+c_{2} .
\end{aligned}
$$

Esta invariancia es denominada invariancia gauge local ya que la constante que se adiciona depende de cada observador al realizar su propia recalibración.

Movimiento circular. El movimiento circular de una partícula en el plano puede ser descrito a partir de la idea gauge. En este caso, el estado de la partícula en cualquier instante queda determinado por su posición y momentum. La posición de la partícula se fija haciendo uso de un plano cartesiano especificando la posición angular por el ángulo que forma con una dirección del plano como se muestra en la figura 1.

La posición angular inicial de la partícula en $\left(P_{1}\right)$ quedará descrita por el ángulo $\left(\theta_{1}\right)$ y su posición final en $\left(P_{2}\right)$ queda dada por el ángulo $\left(\theta_{2}\right)$ respecto al eje de referencia $(x)$, en otras palabras, la posición angular $(\theta)$ de la partícula en cualquier instante queda descrita por la siguiente ecuación de movimiento, en donde el número cinco es una elección que se hace de manera arbitraria para ejemplificar la idea:

$$
\theta(t)=5 t^{2} .
$$




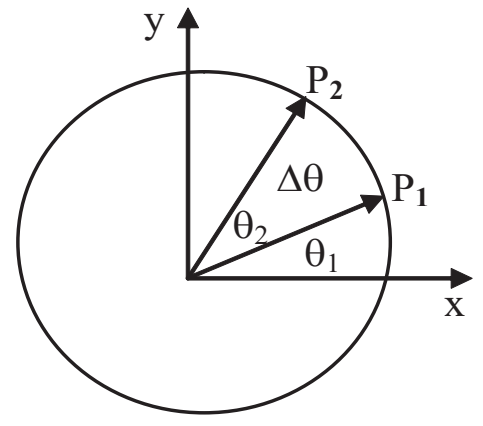

Figura 1. Posición angular de una partícula en un plano cartesiano.

La posición angular de referencia puede ser escogida sin modificar la ecuación de movimiento anterior. Ya que existe una arbitrariedad en la definición de la posición angular de la partícula, se puede introducir el concepto de calibración adicionando una constante arbitraria respecto a la posición angular inicial $\left(\theta_{1}\right)$ y final $\left(\theta_{2}\right)$ dada como:

$$
\begin{aligned}
& \theta_{1} \rightarrow \theta_{1}^{\prime}=\theta_{1}+\text { cte } ; \\
& \theta_{2} \rightarrow \theta_{2}^{\prime}=\theta_{2}+\text { cte } .
\end{aligned}
$$

Lo anterior significa que la elección del punto de referencia angular no tiene importancia.

Por otro lado, para obtener la rapidez angular $(\omega)$ de la partícula en un instante de tiempo $\left(t_{0}\right)$, es necesario conocer la distancia angular, $\theta_{0} \rightarrow \theta^{\prime}=\theta_{0}+\Delta \theta$ que recorre en el instante de tiempo, $t_{0} \rightarrow t^{\prime}=t_{0}+\Delta t$ siendo ( $\Delta t$ ) muy pequeño para evitar cambios en la rapidez de la partícula. En otras palabras, considerando el tiempo y la posición angular de la partícula como una recalibración dada por,

$$
\begin{aligned}
& t_{0} \rightarrow t^{\prime}=t_{0}+\Delta t ; \\
& \theta_{0} \rightarrow \theta^{\prime}=\theta_{0}+\Delta \theta,
\end{aligned}
$$

se obtiene, la posición angular de la partícula un poco más lejos de lo que se encontraba antes $\left(\theta_{0}\left(t_{0}\right)=5 t_{0}^{2}\right)$, es decir, la nueva posición de la partícula será:

$$
\begin{aligned}
\theta^{\prime} & =5 t^{\prime 2}, \\
\theta_{0}+\Delta \theta & =5\left(t_{0}+\Delta t\right)^{2},
\end{aligned}
$$

con respecto a la anterior, $\theta_{0}\left(t_{0}\right)=5 t_{0}^{2}$. Realizando el álgebra se obtiene:

$$
\Delta \theta=10 t_{0} \Delta t+\Delta t^{2},
$$

correspondiendo a la distancia angular adicional recorrida. En primera aproximación la rapidez angular de la partícula estará dada por:

$$
\omega=\frac{\Delta \theta}{\Delta t}=10 t_{0}+\Delta t
$$

Sin embargo, la velocidad angular instantánea es el valor de este cociente cuando $\Delta t$ se hace infinitamente pequeño,

$$
\omega=\lim _{\Delta t \rightarrow 0} \frac{\Delta \theta}{\Delta t}=10 t_{0}
$$

La rapidez angular de la partícula fue obtenida haciendo uso de las transformaciones (7) correspondiendo a una recalibración de las variables de posición angular $\theta=\theta(t)$ y tiempo $(t)$.

El anterior análisis permite caracterizar el papel que juega el concepto gauge en la formalización y explicación del movimiento de una partícula en rotación, lo cual da la posibilidad de mostrar una mirada diferente a la usualmente establecida. Además, la obtención de la rapidez angular de la partícula puede ser mirada como la derivada de la ecuación de posición angular $\theta_{0}\left(t_{0}\right)=5 t_{0}^{2}$ con respecto al tiempo. En este sentido, la implementación de la idea de recalibración en las variables de cualquier función permite obtener la derivada de la función.

Movimiento de rotación de una partícula haciendo uso del plano complejo. El análisis sobre el movimiento de una partícula en rotación desde un sistema de referencia $\left(0^{\prime}\right)$, se realiza considerando la rotación pura del sistema sin que éste tenga translación respecto a un sistema de referencia inercial (0). Los orígenes de los dos sistemas de referencia coinciden y de igual forma sus ejes $z, z^{\prime}$. La velocidad angular del sistema rotante se considera a lo largo del eje común $z, z^{\prime}$, $\mathrm{y}$ en el instante de tiempo $(t)$ los ejes $x, x^{\prime}$, coinciden. El vector de posición de la partícula respecto a los sistemas de referencia $\left(0^{\prime}\right)$ y $(0)$ está dado por:

$$
\vec{r}=x \hat{e}_{1}+y \hat{e}_{2} ; \vec{r}=x^{\prime} \hat{e}_{1}^{\prime}+y^{\prime} \hat{e}_{2}^{\prime},
$$

siendo $\hat{e}_{1}, \hat{e}_{2}, \hat{e}_{1}^{\prime}, \hat{e}_{2}^{\prime}$, los vectores unitarios de cada base coordenada.

Sin embargo, para la descripción del movimiento de la partícula resulta conveniente utilizar un vector rotatorio respecto a los ejes de coordenadas definido por (French, 1974):

$$
\vec{r}=x+i y,
$$

cuyas componentes pueden ser escritas como,

$$
x=r \cos \theta ; y=r \sin \theta,
$$

sin perder información. Además, se puede prescindir de la notación vectorial utilizando el plano complejo para introducir la magnitud $(z)$ que se identifica con la definición de $\vec{r}$ dada por,

$$
\vec{r} \rightarrow z=x+i y,
$$

como se muestra en la figura 2 .

En términos geométricos, la ecuación (13) describe una rotación continua del vector $(z)$ respecto de un eje. Resulta conveniente introducir además la función (French, 1974),

$$
e^{i \theta}=\cos \theta+i \sin \theta,
$$

que representa una rotación de valor $(\theta)$ del vector representado por $(z)$. Esta representación, de un vector por un número complejo, permite describir el movimiento de rotación de la partícula a partir del vector de posición:

$$
\begin{gathered}
z=r e^{\mathrm{i} \theta}, \\
\dot{\mathrm{o}} \\
z=e^{\mathrm{\theta} \theta} \operatorname{con}, r=1,
\end{gathered}
$$

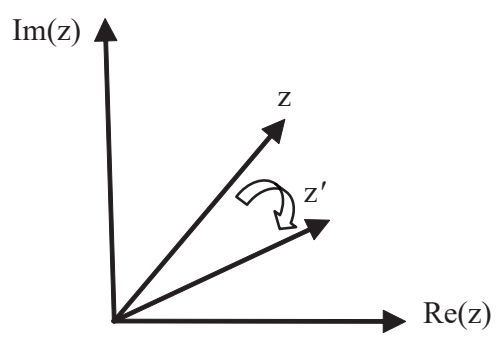

Figura 2. Representación compleja del vector posición. 
donde los números $\left(1, e^{\mathrm{i} \theta}, e^{-\mathrm{i} \theta}\right)$ forman un grupo cuya estructura general es de la forma $\left\{e, a, a^{2}, \ldots, a^{n-1}\right\}$ con $(n)$ cualquier entero positivo (Tung, 2005). Escribir la posición de la partícula en términos de $(z)$ en el plano complejo, permite implementar el concepto de invariancia gauge en la ecuación de posición de la partícula.

Ya que la posición angular $(\theta)$ de la partícula no está unívocamente definida, existe la posibilidad de elegir el ángulo de referencia inicial de una manera arbitraria tal como:

$$
\theta \rightarrow \theta^{\prime}=\theta-\alpha
$$

lo cual significa hacer una redefinición o recalibración (Weyl, 1918) y (Quigg, 1993) de la posición angular de la partícula como se muestra en la figura 3

La ecuación de posición de la partícula no cambia bajo la transformación,

$$
z \rightarrow z^{\prime}=e^{-\mathrm{i} \alpha} z
$$

siendo $(\alpha)$ una constante real e igual para todos los observadores, con los números $\left(1, e^{\mathrm{i} \alpha}, e^{-\mathrm{i} \alpha}\right)$ formando una estructura de grupo.

La derivada de la posición de la partícula transforma como:

$$
\frac{d z^{\prime}}{d t}=e^{-i \alpha} \frac{d z}{d t}
$$

siendo su magnitud invariante,

$$
\left|\frac{d z^{\prime}}{d t}\right|=\left|\frac{d z}{d t}\right| .
$$

Considerando la rapidez de la partícula constante, la aceleración de la partícula queda dada por:

$$
\frac{d^{2} z^{\prime}}{d t^{2}}=e^{-i \alpha} \frac{d^{2} z}{d t^{2}}
$$

siendo también invariante. Asumiendo la masa $(m)$ de la partícula invariante, la fuerza centrípeta será igual para todos los observadores inerciales,

$$
\vec{F}_{c}=\vec{F}_{c}^{\prime} \text {. }
$$

Por otro lado, la recalibración de la posición de la partícula se puede realizar también haciendo depender la constante $(\alpha)$ del tiempo $\alpha=\alpha(t)$. Haciendo uso de la dependencia temporal de la fase $\alpha=\alpha(t)$, la transformación (17) puede ser escrita como:

$$
z \rightarrow z^{\prime}=e^{-i \alpha(t)} z
$$

La derivada de la ecuación de posición de la partícula en este caso transforma de la forma,

$$
\frac{d z^{\prime}}{d t}=e^{-i \alpha(t)}\left(-i z \frac{d \alpha}{d t}+\frac{d z}{d t}\right)
$$

la cual no transforma como se muestra en la ecuación (18), obteniéndose una no invariancia. Sin embargo, la invariancia se recobra remplazando la derivada común $\frac{d}{d t}$ por la derivada covariante (Quigg, 1993),

$$
\frac{d}{d t} \rightarrow \frac{d^{\prime}}{d t}=\frac{d}{d t}+i A(t)
$$

ó,

$$
\left.D \rightarrow D^{\prime}=D+i A(t)\right)
$$

siendo $A=A(t)$ un parámetro compensador (Quigg, 1993) dependiente del tiempo. Por lo tanto, haciendo uso de la derivada covariante la transformación de la derivada de la posición de la partícula queda dada por:

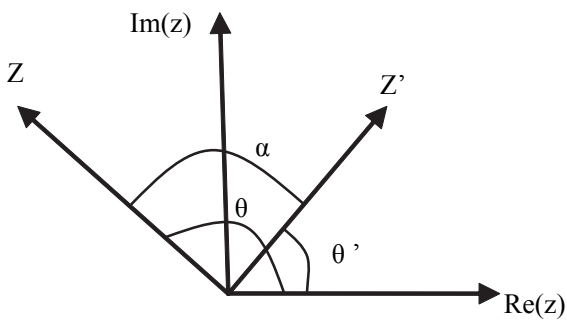

Figura 3. Recalibración de la posición angular $(\theta)$ del vector representado por $(z)$.

$$
\frac{d^{\prime} z^{\prime}}{d t}=e^{-i \alpha(t)} \frac{d z}{d t}
$$

sí,

$$
A(t)=\frac{d \alpha}{d t},
$$

con dimensiones de velocidad angular $A(t)=\omega(t)$. De esta forma, la exigencia de la invariancia de la derivada de la ecuación de posición conduce a la introducción de un término compensatorio $A(t)$ a través de la derivada covariante; termino que es interpretado como la velocidad angular de la partícula. Por lo tanto, la utilización del plano complejo para la determinación de la posición de la partícula, da la posibilidad de introducir el concepto de invariancia gauge en la descripción del movimiento de la partícula en rotación. La velocidad de la partícula queda definida entonces por:

$$
v=D z=\frac{d z}{d t}+i \frac{d \alpha}{d t} z
$$

siendo el término $\operatorname{Re}(v)=\frac{d z}{d t}$ la componente radial de la velocidad, y el término $\operatorname{Im}(v)=\frac{d \alpha}{d t} z$ la componente de la velocidad en la dirección tangencial de la partícula. La aceleración se encuentra diferenciando la velocidad de la partícula,

$$
a=(D v)=\left(\frac{d}{d t}+i A(t)\right)\left(\frac{d z}{d t}+i \frac{d \alpha}{d t} z\right)
$$

ó,

$$
a=\ddot{z}-z \dot{\alpha}^{2}+i(z \ddot{\alpha}+2 \dot{z} \dot{\alpha}) .
$$

En la parte $\operatorname{Re}(a)=\ddot{z}-z \dot{\alpha}^{2}$, el término ( $\left.\ddot{z}\right)$ corresponde a la aceleración en la dirección radial debida al cambio de la rapidez radial, y el término $\left(-z \dot{\alpha}^{2}\right)$ corresponde a la aceleración centrípeta debida al cambio del vector velocidad tangencial. En la parte $\operatorname{Im}(a)=z \ddot{\alpha}+2 \dot{z} \dot{\alpha}$, el término $(z \ddot{\alpha})$ da la aceleración de la partícula en la dirección tangencial debida al cambio en la magnitud de la velocidad tangencial, y el término $(2 \dot{z} \dot{\alpha})$ representa la aceleración de coriolis, la cual en parte es debida al cambio en la rapidez radial y al cambio de la rapidez tangencial.

En la literatura (Fetter, et al., 1980) y (Kleppner, et al., 1973) se encuentra la aceleración de la partícula escrita en coordenadas polares como:

$$
\vec{a}=\left(\ddot{r}-r \dot{\theta}^{2}\right) \hat{r}+(r \ddot{\theta}+2 \dot{r} \dot{\theta}) \hat{\theta},
$$

cuya forma corresponde a la ecuación (29). Finalmente, los efectos dinámicos que obran sobre la partícula son descritos por las fuerzas, 
Tabla 1. Cuadro comparativo sobre el movimiento de una partícula en rotación haciendo uso de coordenadas polares y del plano complejo

\begin{tabular}{|c|c|}
\hline Coordenadas polares & Plano complejo \\
\hline Posición: & Posición: \\
\hline$\vec{r}=r \hat{r}$ & $z=x+i y$ \\
\hline Velocidad: & Velocidad: \\
\hline$\vec{v}=\dot{r} \hat{r}+r \dot{\theta} \hat{\theta}$ & $v=D z=\frac{d z}{d t}+i z \frac{d \alpha}{d t}$ \\
\hline Aceleración: & Aceleración: \\
\hline$\vec{a}=\left(\ddot{r}-r \dot{\theta}^{2}\right) \hat{r}+(r \ddot{\theta}+2 \dot{r} \dot{\theta}) \hat{\theta}$ & $a=\ddot{z}-z \dot{\alpha}^{2}+i(z \ddot{\alpha}+2 \dot{z} \dot{\alpha})$ \\
\hline Fuerza: & Fuerza: \\
\hline$\vec{F}=m\left(\ddot{r}-r \dot{\theta}^{2}\right) \hat{r}+m(r \ddot{\theta}+2 \dot{r} \dot{\theta}) \hat{\theta}$ & $F=m a=m\left(\ddot{z}-z \dot{\alpha}^{2}\right)+i m(z \ddot{\alpha}+2 \dot{z} \dot{\alpha})$ \\
\hline$\vec{F}_{\text {centrifuga }}=m \ddot{r} \hat{r} ; \vec{F}_{\text {centripeta }}=-m r \dot{\theta}^{2} \hat{r}$ & $F_{\text {centrifuga }}=m \ddot{z} ; F_{\text {centripeta }}=-m z \dot{\alpha}^{2}$ \\
\hline$\vec{F}_{\text {tangencial }}=m r \ddot{\theta} \hat{\theta} ; \vec{F}_{\text {coriolis }}=2 m \dot{r} \dot{\theta} \hat{\theta}$ & $F_{\text {tagencial }}=m z \ddot{\alpha} ; F_{\text {coriolis }}=2 m \dot{z} \dot{\alpha}$ \\
\hline
\end{tabular}

$$
\begin{aligned}
& F_{\text {centrifuga }}=m \ddot{z} ; F_{\text {centripeta }}=-m z \dot{\alpha}^{2}, \\
& F_{\text {tagencial }}=m z \ddot{\alpha} ; F_{\text {coriolis }}=2 m \dot{z} \dot{\alpha},
\end{aligned}
$$

ó,

$$
F=m a=m\left(\ddot{z}-z \dot{\alpha}^{2}\right)+i m(z \ddot{\alpha}+2 \dot{z} \dot{\alpha}) .
$$

El resultado (33) es semejante al dado por (1), cuando se hace un análisis sobre el movimiento de la partícula en rotación haciendo uso de coordenadas polares (Kleppner, et al., 1973). Finalmente se muestra un cuadro comparativo entre el análisis que usualmente se realiza haciendo uso de coordenadas polares y el que se propone haciendo uso del plano complejo introduciendo el concepto de invariancia gauge, como se muestra en la tabla 1.

\section{Conclusiones}

El esquema de la mecánica newtoniana permite hacer una descripción del movimiento de una partícula en rotación respecto a sistemas de referencia arbitrarios. Bajo esta perspectiva, se obtienen los efectos dinámicos; las fuerzas de coriolis, centrifuga, centrípeta y tangencial. Sin embargo, los mismos efectos dinámicos pueden ser obtenidos elaborando una formalización diferente, haciendo uso del plano complejo y a su vez implementando el concepto de invariancia gauge en la ecuación de posición de la partícula.

La idea planteada constituye un aporte original y una forma alternativa de enseñanza para abordar la física de una partícula en rotación dentro de un contexto diferente al usualmente establecido, lo cual permite crear un puente entre el conocimiento adquirido por los estudiantes y las nuevas ideas que utilizan los pensadores para la explicación de los fenómenos.

Este tipo de análisis muestra un aporte novedoso para deducir las ecuaciones dinámicas de una partícula en rotación y constituye una manera conveniente de familiarizar a los estudiantes con temas contemporáneos de la física.
Se plantea una forma diferente y didáctica de abordar el movimiento de una partícula en rotación utilizando procedimientos similares a la construcción de una teoría gauge local.

\section{Agradecimientos}

Este escrito ha sido realizado con el apoyo de la Universidad Pedagógica Nacional.

\section{Conflicto de intereses}

El autor manifiesta que no tiene conflicto de intereses.

\section{Referencias}

Kleppner, D., et al. 1973. An Introduction to Mechanics, McGrawHill, Masachusetts, E.U, pp 27-38.

Feynman, R. 1964. The Feynman lectures on Physics, Vol I, Addison- Wesley Publishing Company, Masachusetts, U.S.A, pp 20-1-12.

Marion, J. B. 1992. Dinámica Clásica de las Partículas y Sistemas. Reverté S.A. España, pp 387-393.

Goldstein, H. 1987. Mecánica Clásica, Editorial Reverté S.A. España, pp 226-232.

Greiner, W., et al. 2000. Gauge Theory of Weak Interactions, Springer, New York, pp 107-168.

Quigg, C. 1993. Gauge Theories of the Strong, Weak, and Electromagnetic Interactions, Frontiers in Physics Addison-Wesley, Canada, pp 37-43.

Novaes, S. 2000. Standard Model: An Introduction. Instituto de Física Teórica, Universidade Estadual Paulista, Brazil. arXiv:hep-ph/0001283 v1 Jan 2000.

Quigg, C. 2015. Electroweak Symmetry Breaking in Historical Perspective arXiv:1503.01756v3, 11 de Mayo 2015.

Weyl, H. 1918. Gravitation and Electricity, Ann. Phys. 59, p 101.

Weyl H. 1952. Space Time Matter, Dover Publications INC, New York, pp 282-295.

French, A. 1974. Vibraciones y Ondas, Editorial Reverté S. A, España, pp 8-18.

Tung, W. 2005. Group Theory in Physics, Word Scientific Publishing Co. Pte. Ltd, pp 12-30.

Fetter, A. L., et al. 1980. Theorical Mechanics of Particles and Continua, McGraw-Hill, U.S.A, pp 31-37. 